\title{
BMJ Open Cohort profile: the Alberta Prostate Cancer Research Initiative (APCaRI) Registry and Biorepository facilitates technology translation to the clinic through the use of linked, longitudinal clinical and patient-reported data and biospecimens from men in Alberta, Canada
}

Catalina Vasquez, ${ }^{1}$ Michael Kolinsky, ${ }^{1}$ Rume Djebah, ${ }^{1}$ Maxwell Uhlich, ${ }^{2}$ Bryan Donnelly, ${ }^{3}$ Adrian S Fairey, ${ }^{4}$ Eric Hyndman, ${ }^{5}$ Nawaid Usmani, ${ }^{1}$ Jackson Wu, ${ }^{5}$ Peter Venner, ${ }^{1}$ Dean Ruether, ${ }^{6}$ Gerald Todd, ${ }^{4}$ Michael Chetner, ${ }^{4} \mathrm{R}$ Trafford Crump, ${ }^{3}$ Perrin H Beatty, ${ }^{1}$ John D Lewis (D) ${ }^{1}$

To cite: Vasquez C, Kolinsky M, Djebah R, et al. Cohort profile: the Alberta Prostate Cancer Research Initiative (APCaRI) Registry and Biorepository facilitates technology translation to the clinic through the use of linked, longitudinal clinical and patient-reported data and biospecimens from men in Alberta, Canada. BMJ Open 2020;10:e037222. doi:10.1136/ bmjopen-2020-037222

- Prepublication history for this paper is available online To view these files, please visit the journal online (http://dx.doi. org/10.1136/bmjopen-2020037222).

Received 23 January 2020 Revised 24 August 2020 Accepted 06 September 2020

Check for updates

(c) Author(s) (or their employer(s)) 2020. Re-use permitted under CC BY-NC. No commercial re-use. See rights and permissions. Published by BMJ.

For numbered affiliations see end of article.

Correspondence to Professor John D Lewis; jdlewis@ualberta.ca

\section{ABSTRACT}

Purpose The Alberta Prostate Cancer Research Initiative (APCaRI) Registry and Biorepository was established in 2014 by the APCaRI to facilitate the collection of clinical and patient-reported data, biospecimen, to measure prostate cancer outcomes and to support the development and clinical translation of innovative technologies to better diagnose and predict outcomes for patients with prostate cancer. Participants Men suspected with prostate cancer and referred to Urology centres in Alberta were enrolled in the APCaRI 01 study, while men with a prior prostate cancer diagnosis participated in the APCaRI 03 study from 1 July 2014 to 30 June 2019. The APCaRI Registry and Biorepository links biospecimens and data from a wide representation of patients drawn from an Alberta population of more than 4 million. Findings to date From 1 July 2014 to 30 June 2019, total APCaRI 01 and 03 study recruitment was 3754 men; $142(4 \%)$ of these men withdrew in full, 65 men (2\%) withdrew biospecimens and 123 men (3\%) died of any cause. Over this same time, 8677 patientreported outcome measure (PROM) surveys and 7368 biospecimens were collected and are available from the registry and biorepository, respectively. The data entry error rate was $0.8 \%$ and $0.95 \%$ for critical and non-critical values, respectively, and $1.8 \%$ for patientreported surveys.

Future plans The APCaRI Registry and Biorepository will collect longitudinal data and PROM surveys until 2024, patient outcomes up to 25 years after recruitment and biospecimen storage for up to 25 years. The APCaRI cohorts will continue to provide data and samples to researchers conducting retrospective

\section{Strengths and limitations of this study}

- The participants' healthcare data and donated biospecimens are linked between the Registry and Biorepository that expands the scope of the data to include high-quality clinical data that include age, prostate specific antigen levels, biopsy results, treatments used, treatment outcomes, patientreported outcome measures, comorbidities, disease state conversions, event dates and biospecimens.

- Biospecimens and clinical information are collected both prospectively and longitudinally from potential and confirmed patients with prostate cancer from a region with a population exceeding 4.3 million.

- There are up to 64 sample aliquots per participant per timepoint; including serum, plasma, buffy coat, red blood cells, urine and semen to support multiomics research approaches.

- This study is not a population-based cohort (recruited men represent less than $20 \%$ of the Albertan prostate cancer population); therefore, there is an inherent risk of selection bias limiting the generalisability of findings obtained in this setting to the whole population.

- Attendance at annual follow-up visits is a challenge for the Alberta Prostate Cancer Research Initiative study, negatively affecting the percentage of biospecimens donated every year.

studies. The richness of the data and biospecimens will complement many different research questions, ultimately to improve the quality of care for men with prostate cancer. 


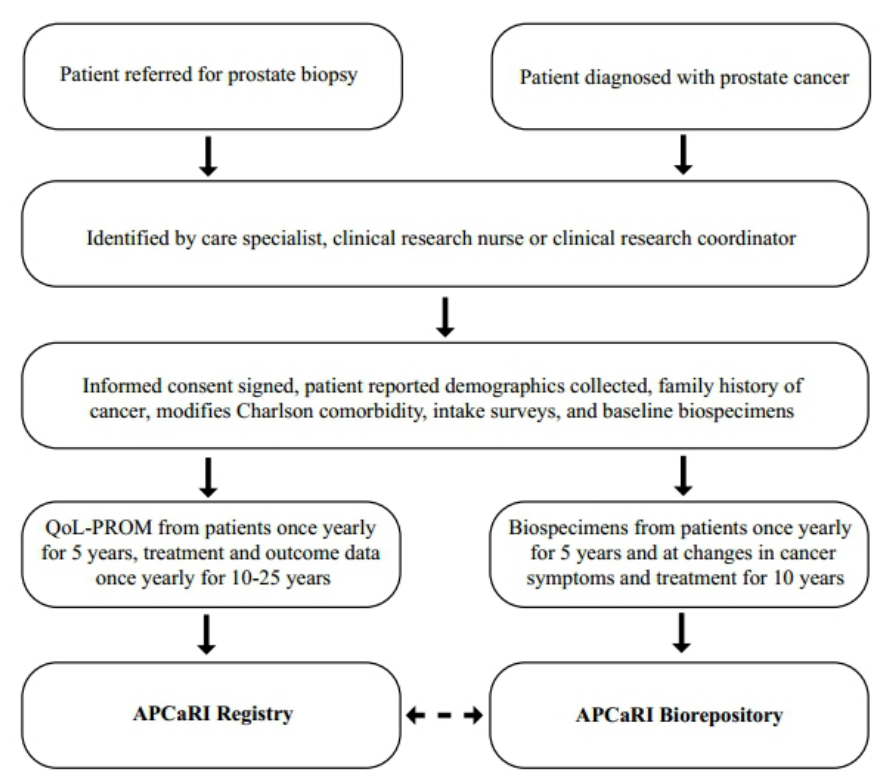

Figure 1 Flow diagram of how patients are recruited for the APCaRI Registry and Biorepository. APCaRI, Alberta Prostate Cancer Research Initiative; PROM, patient-reported outcome measures; QoL, quality of life.

\section{INTRODUCTION}

Globally, prostate cancer (PCa) is the second most common cancer and the second leading cause of cancer deaths for men. ${ }^{1}$ The 5-year overall survival rate for North American men presenting with low risk, localised PCa is $95 \%-99 \%$, but drops to $25 \%-29 \%$ for patients presenting with high risk, metastatic, castrate-resistant prostate cancer. ${ }^{1-3}$ The age-standardised mortality rate has decreased since 1992, when prostate-specific antigen (PSA) testing became available as a laboratory biomarker test. ${ }^{4}$ PSA screening may promote overdiagnosis and a prolonged PCa diagnosis lead-time that mislabels men with an otherwise indolent and non-threatening cancer, leading to overtreatment and reduced quality of life (QoL). ${ }^{5} 6$

Although the US Preventive Services Task Force recommends against population-based PSA screening, uncertainty about lifetime probabilities and misconceptions perpetuated by interest groups, bias clinicians and patients towards it. ${ }^{6}$ There is an unmet clinical need to develop PSA companion biomarker tests to improve PCa diagnostic specificity. Clinically significant PCa biomarkers coupled with patient data, such as age, comorbidities, pathologic indices and others, may provide higher accuracy for early PCa risk-level prognosis. ${ }^{67}$ This would allow targeted clinical care so low-risk patients could avoid unnecessary, potentially harmful, prostate biopsies and treatment, while high-risk patients could undergo immediate treatment. ${ }^{8}$

The research required to understand the disease progression of long-lead time diseases like localised PCa, and to meet the diagnostic and therapeutic clinical care needs, is challenging but greatly improved by access to longitudinal clinical data linked to high-quality stored biospecimens from large patient cohorts of men suspected or diagnosed with PCa. Cross-referenced data and biospecimens allow correlation of risk factors and molecular biomarkers to disease progression and clinical outcome. $^{9}$

The Alberta Prostate Cancer Research Initiative (APCaRI) was established in 2012 as a multidisciplinary research programme with the long-term goals to (1) develop, validate and translate novel non-invasive diagnostics to the clinic to improve prediction of clinically significant PCa and onset of metastasis, (2) integrate research results into standard of care to improve health policy and patient outcomes and (3) apply innovative technologies to the cancer spectrum. To facilitate these goals, APCaRI created the Alberta Prostate Cancer Registry and Biorepository in 2014 to collect, safely store and distribute high-quality linked patient clinical data, patient-reported outcome measure (PROM) surveys and biospecimens both longitudinally and prospectively (figure 1).

The APCaRI Registry is internationally unique due to its comprehensiveness; with patient clinical data collected every 6 months for up to 25 years and PROM and standardised (QoL) survey answers collected yearly for 5 consecutive years. The prospectively collected data are used to meet various study objectives, such as evaluation of QoL outcomes with the PROM surveys and patterns of care across regions in Alberta.

There are numerous examples of successful translation facilitated by these resources including a prospective study aimed to develop an accurate, minimally invasive test to diagnose PCa. The investigators used a subset of samples and clinical data to validate a novel extracellular vesicle (EV) biomarker blood test called ClarityDX Prostate that predicts clinically significant PCa with high accuracy using a platform that combines a microflow cytometry EV biomarker assay, clinical features and machine learning algorithms to calculate a patient risk score and develop an online risk predictor calculator. ${ }^{10}{ }^{11}$ Samples are also supporting research towards development of new tests to predict recurrence for patients on active surveillance and to predict early onset of metastasis post-treatment. Other studies are looking at patient care and adverse effects associated with overtreatment by analysing longitudinal QoL follow-up survey answers in an active surveillance subset of the APCaRI cohort.

The APCaRI Registry is one of the participating sites contributing to the TrueNTH Global Registry, an international registry to monitor PCa care globally aiming to reduce unwarranted variation and to promote universal excellence in the scope and quality of data collection and storage. ${ }^{12}$ The APCaRI Registry began data uploading to the TrueNTH Global Registry in January 2020 and continues to do so currently. The APCaRI Registry collects all the data elements required by the TrueNTH Registry plus an additional 1472 unique fields.

This paper describes the APCaRI Registry and Biorepository initiative, explains the collection procedures for data and biospecimens, lists the measures to ensure 
quality of the data and the biospecimens, reports the profile of the data and biospecimens, outlines the process to access the Registry and lists publications resulted from using this registry. We also describe the challenges of collecting the data and biospecimens and discuss possible solutions to these challenges and issues.

We hope researchers who are planning to do a similar initiative could learn from our experiences and welcome translational researchers to make use of these resources for their studies.

\section{COHORT DESCRIPTION}

Patient recruitment occurs in Edmonton, Canada at the Northern Alberta Urology Centre, Kaye Edmonton Clinic and Cross Cancer Institute; and in Calgary, Canada at the Prostate Cancer Centre and Tom Baker Cancer Centre. Patients are eligible for enrolment in two separate prospective cohort protocols: (1) prediagnosis (APCaRI 01 Protocol, REB approved number HREBA-CC-18-0513) if they have no prior PCa diagnosis and present with either a PSA-screened or clinical suspicion of PCa requiring prostate biopsies or transurethral resection of prostate (TURP) and (2) postdiagnosis (APCaRI 03 Protocol, REB approved number HREBA-CG 14-0085) for participants with known or treated PCa or presenting with initial metastatic PCa.

Consenting patients complete an intake process including clinical collection of demographic information, family history of cancer and the modified Charlson Comorbidity Index Questionnaire (table 1). QoL and PROM surveys are collected annually and include a medication questionnaire, the International Prostate Symptom Score, Expanded Prostate Index Composite, 26 Item, Short Form and the five-level EuroQol FiveDimension Questionnaire. ${ }^{13-15}$ Longitudinal clinical data are collected every 6 months and at changes in cancer symptoms or treatment for 10 years and continuing clinical observations and data may be recorded for up to 25 years (tables 1 and 2).

These data are stored and managed in Research Electronic Data Capture tools hosted and maintained by the University of Alberta. ${ }^{16}$ Rigorous data quality control procedures include: (1) entered data are visually and electronically validated by data entry clerks and the data administrator, (2) each data field is classified as either a critical or non-critical value, (3) all critical values are verified by a team member other than the data entry clerk, while approximately $10 \%$ of the non-critical values are verified bi-annually, (4) data entry error rates and error patterns are reported to the APCaRI science and data quality committee on a bi-annual basis, and the data quality control (QC) is improved accordingly. The APCaRI Registry data are queried, compared, analysed and visualised using the Predictive Research Online System for Prostate Cancer Tasks (PROSPeCT); APCaRI's user-friendly online Clinical Information System. ${ }^{17}$
Blood, urine and/or semen biospecimens are collected at baseline and annually for 5 years as well as at points of PCa disease status changes according to APCaRI standard operating procedures (SOPs; tables 1 and 2). Blood samples are clotted as applicable, centrifuged and aliquoted into serum, plasma, buffy coat and red blood cells. Urine samples are aliquoted and stored and semen samples are left at ambient temperature for 15-45 min to allow liquefaction, then aliquoted and stored. The condition of the samples at the time of storage, such as haemolysed or lipemic blood, haematuric or turbid urine and bloody semen, is recorded on the Sample Collection and Processing Form. All biospecimens are assigned a unique identifier, aliquoted into cryovials and frozen at $-80^{\circ} \mathrm{C}$ within 2 hours of venipuncture time (for blood) or collection (for semen and urine) and remain permanently stored at $-80^{\circ} \mathrm{C}$ in the Canadian Biosample Repository.

All APCaRI Biorepository SOPs follow the regulations and guidelines outlined in the International Conference on Harmonization Good Clinical Practice E6(R2), 2011 NCI Best Practices for Specimen Resources, N2 Canada Network of Networks Biospecimen Related SOPs, TriCouncil Policy Statement, Canadian Tissue Repository Network (CTRNet) SOPs and the Canadian Tumour Repository Network. ${ }^{18-22}$ The APCaRI Biorepository has been awarded certification as a quality-controlled biospecimen bank by CTRNet.

\section{Prediagnosis Cohort: APCaRI 01 Protocol}

From 1 July 2014 to 30 June 2019, a total of 2293 patients referred for prostate biopsy or transrectal ultrasound (TURP) were consented and enrolled to APCaRI 01 prior to biopsy (table 3). APCaRI 01 does include patients who had prerecruitment biopsies; of the 1446 individuals in APCaRI 01 who had a positive biopsy anytime, 76 of these individuals $(3 \%)$ had at least one benign biopsy before their positive biopsy. At baseline, 2264 (99\%) completed the intake survey, $2050(89 \%)$ had an initial body mass index (BMI) value recorded and 2259 (99\%) had a completed PSA value. The median PSA was $6.3 \mathrm{ng} \mathrm{m} / \mathrm{L}$ at intake (range 0.1-3857 $\mathrm{ng} \mathrm{m} / \mathrm{L}$ ). Of these consented patients, 2259 (99\%) underwent a diagnostic prostate biopsy and 1446 (64\%) were found to have a carcinoma of the prostate with a Gleason score of $\geq 6$. Patients diagnosed with PCa received the following not mutually exclusive treatments: active surveillance in 248 (17\%); radical prostatectomy (RRP) in $541(37 \%)$; cryotherapy in $62(4 \%)$; external beam radiation therapy (EBRT) in 287 (20\%); brachytherapy in $108(7 \%)$; chemotherapy in $29(2 \%)$ and androgen deprivation hormonal therapy in $326(23 \%)$.

\section{Postdiagnosis Cohort: APCaRI 03 Protocol}

From 1 July 2014 to 30 June 2019, a total of 1462 patients who had a prior diagnosis of PCa were consented and enrolled to APCaRI 03 (table 4). At baseline, 1438 (98\%) completed the intake survey, $1220(83 \%)$ had an initial BMI value recorded and $1435(98 \%)$ had a completed 
Table 1 Summary of dataset variables

\begin{tabular}{|c|c|c|}
\hline Measure & Details & Source \\
\hline \multicolumn{3}{|l|}{ Intake: baseline data } \\
\hline Demographic information & $\begin{array}{l}\text { Date of birth } \\
\text { Race } \\
\text { Weight and height }\end{array}$ & Patient \\
\hline Family history of cancer & Patient reported & Patient \\
\hline $\begin{array}{l}\text { Modified Charlson comorbidity index } \\
\text { questionnaire }\end{array}$ & Patient reported & Patient \\
\hline
\end{tabular}

\section{Data updated annually for 5 years}

Medication questionnaire Current use of prescribed and overthe-counter medications, vitamins,

supplements, etc.

Smoking history

Fasting status

\begin{tabular}{|c|c|c|}
\hline Patient-reported health status & $\begin{array}{l}\text { IPSS } \\
\text { EPIC-26 SF } \\
\text { EQ-5D-5L }\end{array}$ & Patient \\
\hline Biospecimen information & Collection and processing information & APCaRI team \\
\hline \multicolumn{3}{|c|}{ Diagnosis: clinical data sources are reviewed every 6 months and new data are entered in the APCaRI Registry as applicable } \\
\hline PSA & $\begin{array}{l}\text { Three most recent values before } \\
\text { histological diagnosis }\end{array}$ & Clinical data abstraction \\
\hline Pathology & $\begin{array}{l}\text { Complete pathology report from } \\
\text { biopsy, TURP and/or radical retropubic } \\
\text { prostatectomy } \\
\text { Gleason grade and score } \\
\text { Disease involvement and location } \\
\text { Clinical and pathologic staging } \\
\text { Margin status and invasion }\end{array}$ & Clinical data abstraction \\
\hline Imaging & $\begin{array}{l}\text { Bone scan } \\
\text { MRI } \\
\text { CT scan } \\
\text { Other }\end{array}$ & Clinical data abstraction \\
\hline
\end{tabular}

Treatment: clinical data sources are reviewed every 6 months and new data are entered in the APCaRI Registry as applicable

Active surveillance

Start and end date

Clinical data abstraction

Failure

Reason for failure

Treatment option

\begin{tabular}{|c|c|c|}
\hline Radical prostatectomy & $\begin{array}{l}\text { Date } \\
\text { Type of procedure } \\
\text { Nerve sparing } \\
\text { Surgical time } \\
\text { Blood loss } \\
\text { Complications }\end{array}$ & Clinical data abstraction \\
\hline External beam radiation therapy & $\begin{array}{l}\text { Date } \\
\text { Start and end date } \\
\text { Treatment intent } \\
\text { Goals of care } \\
\text { Type } \\
\text { Technique } \\
\text { RT volume } \\
\text { Dose } \\
\text { Fractions } \\
\text { Radionuclide } \\
\text { Complications }\end{array}$ & Clinical data abstraction \\
\hline Brachytherapy & Date & Clinical data abstraction \\
\hline
\end{tabular}

Continued 
Table 1 Continued

\begin{tabular}{lll}
\hline Measure & Details & Source \\
\hline Cryotherapy & Date & Clinical data abstraction \\
& Type (primary/salvage) & Clinical data abstraction \\
Androgen deprivation therapy & Agent & \\
& Start and end date & \\
& Dosage & Clinical data abstraction \\
Chemotherapy ${ }^{*}$ & Intention of treatment & \\
& Agent & \\
& Line of treatment & \\
& Start and end date & \\
& Dosage & \\
& Number of cycles & Clinical data abstraction \\
& Treatment intent & \\
Clinical trials & Agent & \\
& Line of treatment & \\
& Start and end date & \\
& Dosage & \\
& Number of cycles & \\
& Treatment intent &
\end{tabular}

Survival and disease control: clinical data sources are reviewed every 6 months and new data are entered in the APCaRI Registry as applicable

\begin{tabular}{lll} 
Disease progression & Type of progression & Clinical data abstraction \\
& PSA values and date & \\
& Date & \\
& Castrate-resistance status & Clinical data abstraction \\
Metastasis-free survival & Site & \\
& Date & \\
Cause-specific survival & Skeletal event & Clinical/administration \\
Overall survival & Cause of death & Clinical/administration \\
\hline
\end{tabular}

${ }^{*}$ Chemotherapy includes anti-androgens, hormones, corticosteroids and radioisotopes

APCaRI, Alberta Prostate Cancer Research Initiative; EPIC-26 SF, Expanded Prostate Index Composite, 26 Item, Short Form; EQ-5D-5L, fivelevel EuroQol Five-Dimension Questionnaire; IPSS, International Prostate Symptom Score; PSA, prostate specific antigen; TURP, transurethral resection of prostate.

PSA value. The median PSA was $6.5 \mathrm{ng} \mathrm{m} / \mathrm{L}$ at intake (range $0.0-1189 \mathrm{ng} \mathrm{m} / \mathrm{L}$ ). A pathologic diagnosis of PCa was identified on prior biopsy in 1435 (95\%) with the remainder diagnosed outside of Alberta, without available pathology. These data may be missing for various reasons including that the patient underwent a prostate biopsy in a different health jurisdiction.

These patients had previously received the following, not mutually exclusive, treatments: active surveillance in 419 (29\%), RRP in 417 (29\%), cryotherapy in 99 (7\%),

Table 2 APCaRI registry framework

\begin{tabular}{|c|c|c|c|c|c|}
\hline & Intake & 5 years & $\begin{array}{l}\text { Annually } \\
\text { for: } 10 \text { years }\end{array}$ & $\begin{array}{l}\text { At changes } \\
\text { in cancer } \\
\text { symptoms or } \\
\text { treatment for } \\
10 \text { years }\end{array}$ & $\begin{array}{l}\text { Continuing } \\
\text { observations up to } \\
25 \text { years }\end{array}$ \\
\hline Initiation consent form signed & $\mathrm{X}$ & & & & \\
\hline Intake survey & $x$ & & & & \\
\hline Biospecimen collection & $x$ & $X$ & & $\mathrm{X}$ & \\
\hline Longitudinal health information & $\mathrm{X}$ & & $\mathrm{X}$ & $\mathrm{X}$ & $\mathrm{X}$ \\
\hline
\end{tabular}

APCaRI, Alberta Prostate Cancer Research Initiative. 


\begin{tabular}{lll}
$\begin{array}{l}\text { Table } 3 \text { Participants in APCaRI } 01 \\
\text { of prostate cancer }\end{array}$ & \\
\hline $\begin{array}{l}\text { Number of men in 01 protocol who } \\
\text { consented as of 30 June 2019 }\end{array}$ & $\mathbf{2 2 9 3}$ & \\
\hline Prerecruitment biopsy & 76 & $(3 \%)$ \\
\hline Conversions & 65 & $(3 \%)$ \\
\hline Completed intake table & 2264 & $(99 \%)$ \\
\hline Completed BMI value at intake & 2050 & $(89 \%)$ \\
\hline Completed PSA value at intake & 2259 & $(99 \%)$ \\
\hline Mean & 10.78 & \\
\hline Median & 6.3 & \\
\hline Range & $0.1-3857.0$ \\
\hline SD & 86.69 & \\
\hline Biopsy not performed & 34 & $(1 \%)$ \\
\hline Diagnostic prostate biopsy & 2259 & $(99 \%)$ \\
\hline Biopsy positive for malignancy & 1446 & $(64 \%)$ \\
\hline Biopsy negative for malignancy & 813 & $(36 \%)$ \\
\hline Gleason 6 & 410 & $(28 \%)$ \\
\hline Gleason 7 & 880 & $(61 \%)$ \\
\hline Gleason $\geq 8$ & 141 & $(10 \%)$ \\
\hline Missing & 15 & $(1 \%)$ \\
\hline Radical prostatectomy & 541 & $(37 \%)$ \\
\hline Cryotherapy & 62 & $(4 \%)$ \\
\hline Radiation (EBRT) & 287 & $(20 \%)$ \\
\hline Radiation (Brachy) & 108 & $(7 \%)$ \\
\hline Chemotherapy & 29 & $(2 \%)$ \\
\hline Active surveillance & 248 & $(17 \%)$ \\
\hline Androgen deprivation therapy & 326 & $(23 \%)$ \\
\hline
\end{tabular}

APCaRI, Alberta Prostate Cancer Research Initiative; BMI, body mass index; EBRT, external beam radiation therapy; PSA, prostate specific antigen.

EBRT in 433 (30\%), brachytherapy in 99 (7\%), chemotherapy in $50(3 \%)$ and androgen deprivation hormone therapy in $526(37 \%)$.

\section{Patient and public involvement}

Patients participating in this study were not involved in the design, recruitment, conduct or choice of what results to share or when and how to share them to the wider patient community. However, their healthcare professionals asked them whether they would be willing to participate in this study and they were advised as to the level of burden of intervention and the approximate time required to participate, in terms of length of survey response time and the number of potential years of involvement. Patients are encouraged to access study information, researcher profiles and published results on the APCaRI website as well as attend the APCaRI symposia held two times per year and patient representatives are invited to give patient-impact addresses at all meetings and public press releases. The APCaRI Registry
Table 4 Participants in APCaRI 03 recruited postdiagnosis of prostate cancer

\begin{tabular}{ll}
\hline $\begin{array}{l}\text { Number of men in } 03 \text { protocol who } \\
\text { consented as of } 30 \text { June 2019 }\end{array}$ & $\mathbf{1 4 6 2}$ \\
\hline Completed intake table & $1438(98 \%)$ \\
\hline Completed BMI value at intake & $1220(83 \%)$ \\
\hline Completed PSA value at intake & $1435(98 \%)$ \\
\hline Mean & 11.88 \\
\hline Median & 6.5 \\
\hline Range & $0.0-1189.0$ \\
\hline SD & 46.14 \\
\hline Biopsy not performed & $27(2 \%)$ \\
\hline Diagnostic prostate biopsy & $1435(98 \%)$ \\
\hline Gleason 6 & $493(34 \%)$ \\
\hline Gleason 7 & $748(52 \%)$ \\
\hline Gleason $\geq 8$ & $170(12 \%)$ \\
\hline Missing & $24(2 \%)$ \\
\hline Radical prostatectomy & $417(29 \%)$ \\
\hline Cryotherapy & $99(7 \%)$ \\
\hline Radiation (EBRT) & $433(30 \%)$ \\
\hline Radiation (Brachy) & $99(7 \%)$ \\
\hline Chemotherapy & $50(3 \%)$ \\
\hline Active surveillance & $419(29 \%)$ \\
\hline Androgen deprivation therapy & $526(37 \%)$ \\
\hline
\end{tabular}

APCaRI, Alberta Prostate Cancer Research Initiative; BMI, body mass index; EBRT, external beam radiation therapy; PSA, prostate specific antigen.

and Biorepository began due to the fundraising actions of an patient with Albertan PCa and his wife. They identified a need for PCa research to be done in Alberta, to improve diagnosis, treatment and care for Albertan men and their families.

\section{Findings to date}

As of 30 June 2019, the numbers of PROMs in the Registry and donated biospecimens in the Biorepository combined from the pre and postdiagnosis cohorts, categorised by treatment type and disease state, are listed in tables 5 and 6, respectively. For both cohorts, there was close to uniform acquisition of biospecimens and completion of PROMs. PROMs' collection was slightly higher than biospecimen collection because patients were given the option to answer the PROMs online and so could submit their data from other locales.

Verification of data entry accuracy was assessed by performing visual verification of accuracy and completeness against source documents. Critical values including all data elements associated with the informed consent such as personal health number, date of birth, first and last name, address, phone number, email address and pathology accession number(s) were reviewed. Identified mistakes were corrected to reach an error level of 
Table 5 Number of men consented to the APCaRI 01 and 03 protocols (1 July 2014 to 30 June 2019) who donated biospecimens and PROM data to the APCaRI Biorepository and Registry respectively, at baseline and years 1-4, categorised by therapy type

\begin{tabular}{|c|c|c|c|c|c|c|}
\hline & $\begin{array}{l}\text { Active } \\
\text { surveillance }\end{array}$ & $\begin{array}{l}\text { Radical } \\
\text { prostatectomy }\end{array}$ & $\begin{array}{l}\text { Radiation } \\
\text { therapy }\end{array}$ & $\begin{array}{l}\text { Hormone } \\
\text { treatment }\end{array}$ & Cryotherapy & $\begin{array}{l}\text { Total } \\
\mathbf{n}=\end{array}$ \\
\hline Number of patients & 467 & 958 & 725 & 697 & 154 & 3001 \\
\hline $\begin{array}{l}\text { Biospecimens year } \\
1-4\end{array}$ & 543 & 1274 & 750 & 706 & 229 & 3502 \\
\hline $\begin{array}{l}\text { Disease progression } \\
\text { biospecimens } \\
\text { available }\end{array}$ & 167 & 188 & 63 & 155 & 55 & 628 \\
\hline
\end{tabular}

APCaRI, Alberta Prostate Cancer Research Initiative; PROM, patient-reported outcome measures; QoL, quality of life.

$0 \%$. Non-critical values include all other values that are not in the list of critical values. The number of participants to sample was equal to the square root plus one $(\sqrt{ }+1)$ of the total participants recruited into APCaRI. ${ }^{23}$ Participants' charts are randomly selected for visual verification of non-critical values by data entry clerks. Of 30 June 2019, the APCaRI Registry clinical data entry error rates of $0.8 \%-0.95 \%$ are lower or similar to other clinical data repositories that have reported error rates of $0.5 \%$ to $6.4 \% .^{24}$ The error rate for APCaRI PROMs data entry was $1.8 \%$, which is similar to the rate reported for the data entry of patient-reported outcome questionnaires of $1.01 \%-2.02 \% .^{25}$ The slight increase in error rate for the PROMs is a result of patients' refusal to answer some of the questions. Blank fields are then counted as errors. The low error rates like the ones reported here highlight

Table 6 Number of men consented to the APCaRI 01 and 03 protocols (1 July 2014 to 30 June 2019) who donated biospecimens and PROM data to the APCaRI Biorepository and Registry respectively, at baseline and years 1-4, categorised by disease state

\begin{tabular}{llcl}
\hline & $\begin{array}{l}\text { Advanced } \\
\text { disease* }^{*}\end{array}$ & Death & $\begin{array}{l}\text { Total } \\
\mathbf{n =}\end{array}$ \\
\hline Number of patients & 340 & 144 & 484 \\
\hline Baseline biospecimens & 333 & 139 & 472 \\
\hline Biospecimens year 1-4 & 357 & 79 & 436 \\
QoL baseline & 325 & 133 & 458 \\
\hline QoL year 1-4 & 441 & 93 & 534 \\
\hline
\end{tabular}

${ }^{*}$ Advanced disease: includes metastatic disease and castration resistant prostate cancer.

APCaRI, Alberta Prostate Cancer Research Initiative; PROM, patient-reported outcome measures; QoL, quality of life. the dataset quality and accuracy and give end-users confidence in the integrity of the data. ${ }^{24} 26$

Participants are given the option to withdraw from the study in different instances: (1) terminate ongoing biospecimen and health information collection, including any contact from study coordinator or nurse, (2) terminate only ongoing heath data collection or (3) terminate only ongoing biospecimen collection. For the reporting period, a total of $142(4 \%)$ participants withdrew participation in the study selecting optional and 65 subjects (2\%) chose option 3. As of 30 June 2019, 123 (3\%) participants passed away (table 7).

\section{Strengths and limitations}

Key strengths of the APCaRI Registry and Biorepository are listed below. The APCaRI Registry contains patient clinical data that is updated every 6 months and PROM and standardised QoL survey answers collected yearly. The APCaRI Biorepository contains high-quality serum, plasma, buffy coat, red blood cells, urine and semen biospecimens stored at $-80^{\circ} \mathrm{C}$ following international biospecimen storage and technical guidelines and recommendations. Biospecimens and clinical information are collected both prospectively and longitudinally from potential and confirmed patients with PCa. The participants' healthcare data and donated biospecimens are linked between the Registry and Biorepository that expands the scope of the data to include high-quality biological data and clinical data that include age, PSA levels, treatments used, treatment outcomes, biopsy results, PROM responses, comorbidities, disease state conversions and event dates to allow for rate calculations to be performed.

Key limitations in the APCaRI Registry and Biorepository are listed below. The study was designed to be opt-in, 
Table 7 Number of men recruited, withdrew (either fully, or data only or samples only) or deceased from the APCaRI 01 and 03 protocols between 1 July 2014 and 30 June 2019

\begin{tabular}{lclllc}
\hline & Recruited & Withdrawn (full) & Withdrawn (data) & Withdrawn (samples) & Deceased \\
\hline 1 July 2014 to 30 June 2015 & 621 & 0 & 0 & 0 & 2 \\
1 July 2015 to 30 June 2016 & 1208 & 5 & 0 & 4 & 11 \\
1 July 2016 to 30 June 2017 & 1035 & 34 & 0 & 21 & 30 \\
1 July 2017 to 30 June 2018 & 708 & 60 & 0 & 20 & 38 \\
1 July 2018 to 30 June 2019 & 182 & 43 & 0 & 20 & 42 \\
Total & 3754 & $142(4 \%)$ & $0(0 \%)$ & $65(2 \%)$ & $123(3 \%)$ \\
\hline
\end{tabular}

APCaRI, Alberta Prostate Cancer Research Initiative.

where participants must provide informed consent to be enrolled and thus it is not a population-based cohort. Recruited men represent less than $20 \%$ of the Albertan PCa population. Therefore, there is an inherent risk of selection bias limiting the generalisability of findings obtained in this setting to the whole population. However, the level of selection bias risk may vary depending on the health jurisdiction that the population is within. For example, the percentage of men recruited into the APCaRI 01 cohort who were then diagnosed with PCa after biopsy (64\%) is similar to the percentage of men in the general Albertan population who are diagnosed with PCa after biopsy (approximately 60\%), suggesting that the APCaRI cohort is representative of the general population. Other health jurisdictions though, such as the USA, UK and Australia, report percentages of men diagnosed with PCa after an initial biopsy, triggered by a $\geq 4 \mathrm{ng} / \mathrm{mL}$ PSA level, to be as low as $20 \%-30 \%$. $^{327} 28$ This coincides with current information that PSA levels alone correctly identify PCa only about $25 \%-30 \%$ of the time and that PSA screening promotes unnecessary biopsies. ${ }^{3}$ In Alberta, patient referral to a urological oncologist and a possible biopsy is triggered by a highly abnormal digital rectal exam (DRE), rapidly rising PSA levels or a PSA doubling time within 2 years or less. ${ }^{29} 30$ Therefore, the decision to biopsy due to suspicion of PCa is tempered with strong clinical evidence that positively correlates with the high percentage of biopsied men who are found diagnosed with PCa in Alberta.

Attendance at annual follow-up visits is a challenge for the APCaRI study, negatively affecting the percentage of biospecimens donated every year. Many of the participants leave Alberta during the winter months making timely assessment of long-term biospecimens and QoL seasonally difficult. The present study is observational in nature and although statistical modelling was applied to increase the validity of results obtained, unmeasured confounding variables and selection biases remain legitimate threats to the validity of study findings. Available data sources for the present Registry contain data originally collected for medical care or billing purposes and not for research. In some instances, diagnosis and/or procedure codes may be incomplete and even inaccurate in these source documents resulting in the need to (1) increase human resources to access multiple databases and collect the required elements, (2) establish more stringent quality control measures to ensure data accuracy and (3) changes in staging and grading classification systems may occur during the long-term follow-up period that is required in the present study, resulting in misclassification of some variables over time. These issues should be considered when planning investigations using this data.

\section{Collaboration access to biospecimens and data}

The APCaRI Science Committee, consisting of designated APCaRI members, meets ad hoc to process requests for biospecimens and/or deidentified data samples from principal investigators (PIs). APCaRI member methodological experts are consulted, when appropriate, to discuss application relevance and integrity. Applications require a study-specific statistical analysis plan and undergo review by an APCaRI member statistician. Non-member PI biospecimen and/or data applications must include (1) home-institution research ethics board approval, (2) APCaRI Science Committee approval, (3) research protocol, (4) funding details, (5) PI's curriculum vitae, 6) signed data and/or Material Transfer Agreement and (7) agreement to acknowledge APCaRI in publications and presentations that contain results or information associated with the requested materials. On approval and when applicable, the biospecimens are shipped to the requesting laboratory, using the appropriate packaging and conditions to retain stability. A researcher can request to access the Registry data and Biorepository biospecimens separately.

From the creation of the Registry and Biorepository in mid-2014, APCaRI has shared nearly 6000 biospecimen aliquots and clinical information with academic researchers from Alberta (Edmonton and Calgary), Ontario (Toronto, London), Quebec (Montreal), USA, Finland, China and Germany and with industry researchers in Canada and the USA. All requests for data and biospecimens met the criteria of the registry and biorepository and were granted.

To date, numerous publications have resulted from research supported by the APCaRI registry and biorepository that include an article describing the computer 
interface called PROSPeCT designed for simplified registry queries, the inclusion of registry data into the TrueNTH Global Registry, development of a multiomics biomarker pipeline from seminal plasma of patients with PCa and an analysis of the accuracy of PROM surveys. ${ }^{12} 173132$ Several other manuscripts are in preparation and samples and data were also used to support numerous grant applications. In addition, research topics that were proposed by researchers who were granted access to the Biorepository include bone metabolism in metastatic patients with PCa, antibody development, discovery and validation of novel PCa diagnostic, prognostic and predictive molecular biomarkers. Applications to access the Registry involved studies on estimation of cancer conversion rates from indolent/localised to clinically significant or metastatic cancer; evaluation of longterm changes in urologic symptoms and QoL in men with prostate diseases; description of cancer outcomes, including treatment utilisation and adverse effects and disease-related events such as relapses, morbidity and hospitalisations and evaluation of the influence of comorbidities on long-term health risks.

\section{Future perspectives}

Access to high-quality biospecimens and linked longitudinal patient clinical data and health information, including QoL and PROMs, is essential when conducting translational cancer research aimed at improving diagnostic tools leading to personalised therapy. Biospecimen usage in cancer research, as reported in cancer research journals, increased significantly over a 20-year time period and is predicted to continue to increase, alongside larger study cohort sizes. ${ }^{22} 33$ Biobanks are recommended to focus on quality and standardisation of the stored biospecimens. ${ }^{34} 35$

Overall, the Registry and Biorepository have been successful in meeting all objectives; it operates on budget and has funding to continue for 5 more years. The APCaRI Registry and Biorepository will continue to recruit men to the cohort for another 5 years, until 2024 for a potential 6000 recruited patients in total. Then for 5 consecutive years, we will focus on follow-ups of the original cohort.

The Registry and Biorepository data and biospecimens are being used in research that will improve patient care in the future. For example, a clinically useful database query tool called PROSPeCT was designed to help determine patients' responses to various clinical therapies. APCaRI will also continue contributing to initiatives like TrueNTH as well as to validate the novel diagnostic blood tests to predict risk of PCa progression in participants under active surveillance as well as metastatic risk in participants' pretreatment and post-treatment.

The goal remains to improve diagnosis, therapeutic practice and the lives of men with PCa and their families.

\section{Author affiliations}

${ }^{1}$ Department of Oncology, University of Alberta, Edmonton, Alberta, Canada
${ }^{2}$ Department of Computing Science, University of Alberta, Edmonton, Alberta, Canada

${ }^{3}$ Department of Surgery, University of Calgary, Calgary, Alberta, Canada ${ }^{4}$ Department of Surgery, University of Alberta, Edmonton, Alberta, Canada ${ }^{5}$ Department of Surgical Oncology, University of Calgary, Calgary, Alberta, Canada ${ }^{6}$ Department of Oncology, University of Calgary, Calgary, Alberta, Canada

Acknowledgements We thank the men who consented to be part of the APCaRI studies and donated their time and biospecimens to advance the research and development of PCa tests and treatments. We also thank the donors who generously give to fundraising organizations like the Alberta Cancer Foundation, Bird Dogs, Motorcycle Ride for Dad, Movember and Prostate Cancer Canada, University Hospital Foundation and others. We are very thankful to Yuliya Kulchytska, Homeira Parisa Hamayeli Mehrabani and Candace Davies for their help with the Registry datasets. Lastly, we highlight the contributions from the Northern Alberta Urology Centre, the Prostate Cancer Centre at the Southern Alberta Institute of Urology, the Cross Cancer Institute and the Tom Baker Cancer Centre.

Contributors CV, MK, RD, ASF, PV and JDL contributed to the concept and design of the study. CV, MK, RD, MU, BD, EH, NU, JW, PV, DR, GT, MC, RTC, PHB and ASF contributed to the acquisition, analysis and interpretation of the data. All authors have read and approved the final version of the manuscript to be published.

Funding The Alberta Prostate Cancer Registry and Biorepository is funded by The Bird Dogs and the Alberta Cancer Foundation (grant number 26491) and the Prostate Cancer Canada Movember Translation Acceleration (grant TAG2004-03).

\section{Competing interests None declared.}

Patient and public involvement Patients and/or the public were not involved in the design, or conduct, or reporting, or dissemination plans of this research.

Patient consent for publication Not required.

Provenance and peer review Not commissioned; externally peer reviewed.

Data availability statement All data relevant to the study are included in the article. The Alberta Prostate Cancer Research Initiative's prostate cancer registry contains deidentified participant data. Requests for access can be made via https:// apcari.ca/our-research/biorepository/

Open access This is an open access article distributed in accordance with the Creative Commons Attribution Non Commercial (CC BY-NC 4.0) license, which permits others to distribute, remix, adapt, build upon this work non-commercially, and license their derivative works on different terms, provided the original work is properly cited, appropriate credit is given, any changes made indicated, and the use is non-commercial. See: http://creativecommons.org/licenses/by-nc/4.0/.

ORCID iD

John D Lewis http://orcid.org/0000-0002-7734-1204

\section{REFERENCES}

1 Prostate Cancer Treatment $(\mathrm{PDQ} \otimes)$ Board P, ed. Health professional version. Bethesda, MD: US National cancer Institute, 2018.

2 Berglund A, Garmo H, Tishelman C, et al. Comorbidity, treatment and mortality: a population based cohort study of prostate cancer in PCBaSe Sweden. J Urol 2011;185:833-40.

3 Leslie SW, Soon-Sutton TL, Siref LE. Prostate Cancer. StatPearls [Internet. Treasure Island (FL: StatPearls Publishing, 2020.

4 Negoita S, Feuer EJ, Mariotto A, et al. Annual report to the nation on the status of cancer, part II: recent changes in prostate cancer trends and disease characteristics. Cancer 2018;124:2801-14.

5 Loeb S, Bjurlin MA, Nicholson J, et al. Overdiagnosis and overtreatment of prostate cancer. Eur Urol 2014;65:1046-55.

6 Fenton JJ, Weyrich MS, Durbin S, et al. U.S. preventive services Task force evidence syntheses. prostate-specific antigen-based screening for prostate cancer: a systematic evidence review for the US preventive services Task force. Rockville (MD: Agency for Healthcare Research and Quality (US), 2018.

7 Heidenreich A, Bellmunt J, Bolla M, et al. EAU guidelines on prostate cancer. Part 1: screening, diagnosis, and treatment of clinically localised disease. Eur Urol 2011;59:61-71.

8 Moschini M, Carroll PR, Eggener SE, et al. Low-Risk prostate cancer: identification, management, and outcomes. Eur Urol 2017;72:238-49.

9 Huber J, Herpel E, Jakobi $\mathrm{H}$, et al. Two decades' experience with a prospective Biobank for urologic oncology: research, clinical care, and the patients' view. Urol Oncol 2013;31:990-6. 
10 Fairey A, Paproski RJ, Pink D, et al. Clinical analysis of the extracellular vesicle-fingerprint score blood test to refine the prediction of clinically significant prostate cancer and avoid prostate biopsy, 2019.

11 Paproski RJ, Pink D, Vasquez C, et al. Building predictive disease fingerprints using extracellular vesicle microflow cytometry and machine learning, 2019

12 Evans SM, Millar JL, Moore CM, et al. Cohort profile: the TrueNTH Global Registry - an international registry to monitor and improve localised prostate cancer health outcomes. BMJ Open 2017;7:e017006.

13 Wei JT, Dunn RL, Litwin MS, et al. Development and validation of the expanded prostate cancer index composite (EPIC) for comprehensive assessment of health-related quality of life in men with prostate cancer. Urology 2000;56:899-905.

14 EuroQol-a new facility for the measurement of health-related quality of life.. Health policy. 1990/11/05 ED, 1990: 199-208.

15 Charlson ME, Pompei P, Ales KL, et al. A new method of classifying prognostic comorbidity in longitudinal studies: development and validation. J Chronic Dis 1987;40:373-83.

16 Harris PA, Taylor R, Thielke R, et al. Research electronic data capture (REDCap)--a metadata-driven methodology and workflow process for providing translational research informatics support. J Biomed Inform 2009;42:377-81.

17 Cutumisu M, Vasquez C, Uhlich M, et al. Prospect: a predictive research online system for prostate cancer tasks. JCO Clin Cancer Inform 2019;3:1-12.

18 ICHGCP.NET. Good clinical practice, 2019. Available: https://ichgcp. net/ [Accessed July 18 2019].

19 Greenberg B, Christian J, Henry LM, et al. White Paper, addendum to Registries for Evaluating Patient Outcomes: A User's Guide. ed. Rockville, MD: Prepared by L\&M Policy Research, LLC, under Contract No. 290-2014-00004-C, 2018.

20 N2 Canada Network of Networks, Biospecimen Standard Operating Procedures and Quiz. Canadian cancer clinical trials network, 2019.

21 Tri-Council Policy Statement. Ethical conduct for research involving humans: Canadian Institutes of health research. natural sciences and engineering Research Council of Canada and social sciences and humanities Research Council of Canada, 2014.

22 Standard Operaton Procedures. Canadian tissue Repository network, 2019. Available: https://www.ctrnet.ca/resources/operatingprocedures.
23 Ezzelle J, Rodriguez-Chavez IR, Darden JM, et al. Guidelines on good clinical laboratory practice: bridging operations between research and clinical research laboratories. J Pharm Biomed Anal 2008;46:18-29.

24 Hong MKH, Yao HHI, Pedersen JS, et al. Error rates in a clinical data repository: lessons from the transition to electronic data transfer--a descriptive study. BMJ Open 2013;3:e002406.

25 Paulsen A, Overgaard S, Lauritsen JM. Quality of data entry using single entry, double entry and automated forms Processing-An example based on a study of patient-reported outcomes. PLoS One 2012;7:e35087

26 Mays JA, Mathias PC, Patrick C. Measuring the rate of manual transcription error in outpatient point-of-care testing. J Am Med Inform Assoc 2019;26:269-72.

27 Lopez-Corona E, Ohori M, Wheeler TM, et al. Prostate cancer diagnosed after repeat biopsies have a favorable pathologica outcome but similar recurrence rate. J Urol 2006;175:923-8.

28 EAU Guidelines. EAU guidelines office, Arnhem, the Netherlands. ISBN 978-94-92671-01-1, 2018

29 Bell N, Gorber SC, Shane A, et al. Recommendations on screening for prostate cancer with the prostate-specific antigen test. Can Med Assoc J 2014;186:1225-34.

30 Guidelines for the Use. Alberta Medical association of PSA and the early diagnosis of prostate cancer (2006), 2006. Available: http://www.topalbertadoctors.org/TOP/CPG/ProstateCancer/ ProstateCancer.htm [Accessed Sept 18 2019].

31 Drabovich AP, Saraon P, Drabovich M, et al. Multi-Omics biomarker pipeline reveals elevated levels of Protein-glutamine gammaglutamyltransferase 4 in seminal plasma of prostate cancer patients. Molecular \& Cellular Proteomics 1807;8

32 Crump T, Peterson A, Charbonneau C, et al. Evaluating the psychometric properties of the EPIC-26. European Urology Supplements2018;17:e1830.

33 Biospecimen Standard Operating Procedures and Quiz. Canadian cancer clinical trials network, 2019. Available: https://3ctn.ca/page/ biospecimen-standard-operating-procedures-and-quiz.

34 Gliklich RE, Dreyer NA, Leavy MB. Registries for evaluating patient outcomes, 2014. Available: https://www.ncbi.nlm.nih.gov/books/ NBK208616/

35 Verne J, Hounsome L, Kockelbergh R, et al. Improving outcomes from prostate cancer: unlocking the treasure trove of information in cancer registries. Eur Urol 2016;69:1013-4. 